\title{
Traduire
}

Revue française de la traduction

\section{Informatique dématérialisée et logiciels-services : virtualisation de l'environnement de travail du traducteur}

Anne-Marie Robert

\section{(2) OpenEdition \\ Journals}

Édition électronique

URL : http://journals.openedition.org/traduire/246

DOI : $10.4000 /$ traduire.246

ISSN : 2272-9992

Éditeur

Société française des traducteurs

Édition imprimée

Date de publication : 1 juin 2011

Pagination : 14-20

ISSN : 0395-773X

\section{Référence électronique}

Anne-Marie Robert, «Informatique dématérialisée et logiciels-services : virtualisation de

l'environnement de travail du traducteur », Traduire [En ligne], 224 | 2011, mis en ligne le 04 février 2014, consulté le 01 mai 2019. URL : http://journals.openedition.org/traduire/246 ; DOI : 10.4000/ traduire.246 


\title{
Informatique dématérialisée et logiciels-services : virtualisation de l'environnement de travail du traducteur
}

\author{
Anne-Marie Robert
}

Cet article reprend dans les grandes lignes la conférence donnée par l'auteur en nom et représentation de la SFT le 16 octobre 2010, lors du colloque Traduction et ergonomie organisé par le GREMUTS à l'Université Stendhal Grenoble 3.

\section{Introduction}

Les nouvelles technologies disponibles sur le Web 2.0 font apparaitre de nouveaux concepts, ressources informatiques et pratiques professionnelles. L'informatique dématérialisée et les logiciels-services en sont deux manifestations dans tous les secteurs de l'industrie, y compris celui de la traduction.

\section{Fondements de l'informatique dématérialisée}

Le terme anglais cloud computing est apparu aux États-Unis début 2007 à l'occasion du lancement de la première édition du salon Cloud Expo à New York. Plusieurs termes désignent actuellement ce nouveau concept en français : " informatique en nuage ", " informatique dans les nuages ", "infonuagique " ou encore "informatique dématérialisée ". La référence aux nuages (cloud en anglais) vient du fait qu'Internet est souvent représenté sous la forme de nuages dans les schémas qui illustrent l'interconnexion de réseaux, notamment pour en symboliser l'omniprésence virtuelle. Par souci de pragmatisme et de cohérence terminologique, seul le terme " informatique dématérialisée " est employé dans cet article.

Ce nouveau concept d'informatique dématérialisée a été inventé par le cybermarchand américain Amazon en 2006. Après avoir énormément investi dans ses infrastructures pour lancer sa boutique en ligne à la fin des années 1990, Amazon a constaté que celles-ci permettaient d'absorber son pic d'activité au moment des achats de Noël mais restaient inexploitées le reste de l'année. Pour rentabiliser ses infrastructures tout au long de l'année, Amazon a décidé d'en louer temporairement une partie à des tiers. 


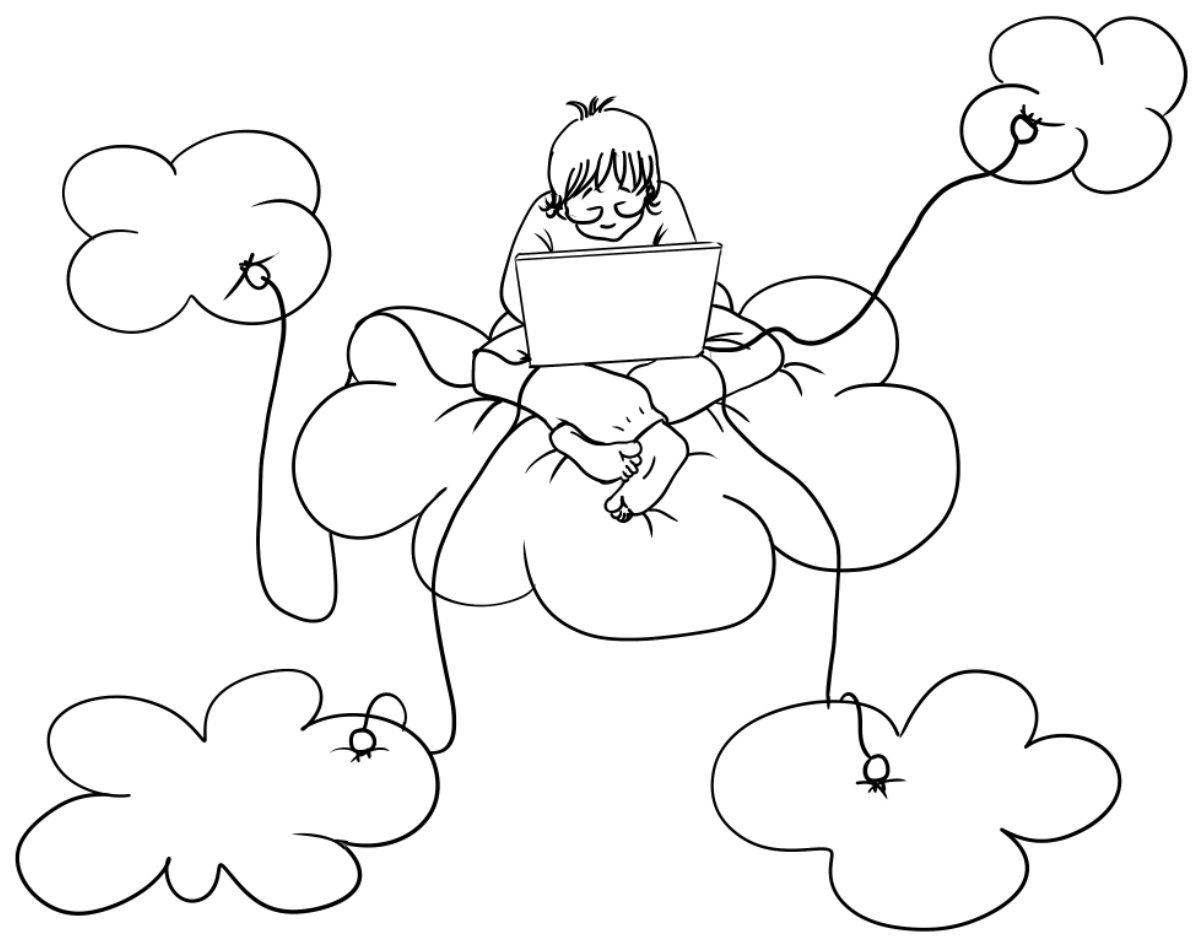

Illustration : Marlène Junius, http://alotoftralala.over-blog.com

L'informatique dématérialisée est un terme générique qui désigne toutes les ressources informatiques (infrastructures, plates-formes, logiciels, services et solutions) uniquement accessibles via Internet. L'environnement informatique ainsi accessible à distance devient donc dé-matérialisé, c'est-à-dire virtuel. Les termes " dématérialisation " et " virtualisation " et leurs dérivés sont souvent employés pour faire référence à ce nouveau concept.

\section{Applications à l'industrie de la traduction}

\subsection{Externalisation informatique}

Un traducteur peut désormais recourir à l'informatique dématérialisée pour externaliser la gestion de tout ou partie de son système informatique. II peut, par exemple, opter pour l'info- 
gérance à distance de son serveur qui ne sera dans ce cas plus physiquement hébergé dans son bureau, mais le sera virtuellement chez un fournisseur extérieur. Il peut également choisir d'effectuer ses sauvegardes informatiques non pas sur un disque dur externe, mais sur un espace de stockage en ligne. Ou encore, il peut décider de " dématérialiser " le circuit de traitement de sa facturation (numérisation, reconnaissance automatique, validation électronique, export des écritures comptables) en passant par un service à la demande en ligne tel que Yooz Business Edition. II peut enfin recourir à des logiciels-services pour gérer son activité (suite bureautique Microsoft Office 365 et messagerie électronique Google Gmail, par exemple).

\subsection{Logiciels-services en traduction}

Les " logiciels-services " ou " logiciels à la demande " (SaaS, Sofware as a Service, en anglais) désignent les logiciels qui sont proposés sous forme de services uniquement accessibles via Internet. L'utilisateur n'achète plus un produit logiciel qu'il installe sur son ordinateur dans le cadre d'une licence, mais il loue l'utilisation à la demande d'un service logiciel uniquement accessible via Internet.

Depuis 2006, le traducteur peut utiliser des logiciels-services spécifiquement développés en ingénierie linguistique pour répondre aux besoins du marché de la traduction. Aucun matériel informatique et aucun personnel de maintenance informatique ne sont désormais nécessaires pour traduire avec des outils informatiques dédiés.

Les logiciels-services en traduction actuellement disponibles via Internet sont aussi divers que variés. Certains s'adressent à des traducteurs amateurs, occasionnels ou professionnels ou encore à des agences de traduction, des entreprises ou des institutions. Certains proposent un environnement de travail professionnel complet ou quelques fonctionnalités parmi lesquelles la gestion de projet, la terminologie, la TAO, la révision ou la prétraduction automatique. Certains sont gratuits tandis que d'autres sont payants. Certains proposent des systèmes hybrides avec centralisation d'outils en ligne et installation d'outils sur son ordinateur. Certains proposent des mémoires de traduction publiques, d'autres des mémoires de traduction privées. Certains sont des logiciels libres.

Les neuf logiciels-services suivants sont présentés avec leurs particularités :

Éditeur : ByteTranslation (Italie) - Produit : Boltran - Public visé : traducteurs occasionnels Fonctionnalité : mémoire de traduction (compatibilité avec le format .tmx) - Particularité : logiciel libre - Gratuit

Éditeur : Crowdin (Ukraine) - Produit : Crowdin - Public visé : localisateurs - Fonctionnalités : TAO avec mémoires de traduction, gestion des flux et outils collaboratifs - Particularité : destiné à la localisation d'interfaces logicielles et d'interfaces Web destinées à la téléphonie mobile - Gratuit 
Éditeur : Google (États-Unis) - Produit : Google Translator Toolkit - Public visé : traducteurs amateurs - Fonctionnalités : mémoire de traduction publique partagée ou création d'une mémoire de traduction non partagée ou partagée uniquement avec des utilisateurs invités (avec possibilité d'importer une mémoire au format .tmx), bases de données terminologiques, messagerie instantanée et prétraduction automatique systématique avec Google Translate Gratuit

Éditeur : GlobalSight Collaborate To Innovate (États-Unis) - Plate-forme : GlobalSight - Public visé : agences de traduction et de localisation, éditeurs de logiciels indépendants, traducteurs et entreprises - Objectifs : communauté de développement collaboratif d'un système de gestion de traduction souple et libre (open source) - Payant : au mois

Éditeur : Lingotek (États-Unis) - Produit : Lingotek - Public visé : agences de traduction et entreprises - Fonctionnalités : plate-forme de traduction collaborative avec mémoire de traduction publique, bases de données terminologiques, gestion de projet et prétraduction automatique avec Google Translate et Microsoft Bing - Payant : au mois

Éditeur : GeoWorkz (États-Unis) - Produit : Translation Workspace - Public visé : traducteurs, agences de traduction et entreprises - Fonctionnalités : TAO avec gestion de projet, conversion, mémoires de traduction, bases de données terminologiques, révision, et assurance qualité - Particularité : système hybride avec centralisation d'outils accessibles uniquement en ligne (mémoires de traduction, bases de données terminologiques, etc.) et installation d'outils sur son ordinateur (barre d'outils spécifique sous Word, logiciel XLIFF Editor pour la prise en charge de fichiers balisés, etc.) - Payant : au mois, avec différents tarifs en fonction du seuil de nombre de mots choisi

Éditeur : Wordbee (Luxembourg) - Produit : Wordbee Translator (une version Freelance et une version Team) - Public visé : traducteurs, agences de traduction et entreprises - Fonctionnalités : TAO avec mémoires de traduction, gestion de projet, bases de données terminologiques, révision, outils collaboratifs et possibilité de prétraduction automatique avec Google Translate - Payant : au semestre ou à l'année

Éditeur : Wordfast LLC (France) - Produit : Wordfast Anywhere - Public visé : traducteurs Fonctionnalités : TAO avec mémoires de traduction et bases de données terminologiques ; mémoire de traduction publique et prétraduction automatique en option - Gratuit

Éditeur : XTM International (Royaume-Uni) - Produit : XTM Cloud (trois versions Freelance, Small Group et LSP) - Public visé : traducteurs et agences de traduction - Fonctionnalités : TAO avec mémoires de traduction, bases de données terminologiques et gestion des flux Payant : au mois, avec différents tarifs en fonction du seuil de nombre de mots choisi 


\section{Conséquences sur la pratique professionnelle}

\subsection{Vers de nouveaux modèles}

Vers un nouveau modèle de distribution en ligne de ressources. Le traducteur passe d'un environnement de travail physique à un environnement de travail virtuel : il n'exerce plus avec ses propres outils installés sur son ordinateur, mais avec des outils numériques accessibles via Internet. II dispose ainsi de ressources non plus statiques, mais dynamiques.

Vers un nouveau modèle économique. Le traducteur passe de l'achat de produits à la location de services. II peut, par exemple, passer de l'achat de licences logicielles à la location de logiciels-services accessibles en ligne, avec tarification à la demande ou à l'utilisation (à l'heure, au mois, à l'année, au giga-octet consommé, au volume de mots, à l'utilisateur, etc.).

Vers un nouveau modèle de gestion informatique. Le traducteur passe d'une gestion manuelle individuelle chronophage à une gestion entièrement automatisée et transparente de ses ressources informatiques. Celles-ci sont accessibles en temps réel, en tout lieu et à tout moment sans que le traducteur ait à se préoccuper de leur gestion, de leur maintenance ni de leur mise à jour ou à niveau.

Vers un nouveau modèle de travail collaboratif. La plupart des nouveaux logiciels-services proposent des fonctionnalités de travail en mode collaboratif (en partageant des mémoires de traduction, en mettant des bases de données terminologiques à jour de façon dynamique, en partageant des informations centralisées, en utilisant un système de messagerie instantanée intégré pour demander conseil, etc.). Le traducteur passe d'un mode de travail individuel et parfois solitaire à un mode de travail collaboratif virtuel qui lui permet d'appartenir à une communauté à l'échelle internationale. II est ainsi en mesure de mutualiser et de partager des connaissances, des compétences et des ressources en vue d'améliorer son efficacité au travail, y compris au sein d'équipes virtuelles dans le cadre de gros projets multilingues.

\subsection{Vers de nouvelles pratiques professionnelles}

La dématérialisation de l'environnement technologique du traducteur amène inexorablement à de nouvelles pratiques. La virtualisation progressive de l'environnement de travail du traducteur passera nécessairement par une phase de transition. Sa capacité d'adaptation l'aidera à prendre ce nouveau virage technologique et à ajuster en conséquence ses pratiques en fonction de ces nouveaux modèles. 


\section{Conclusion}

L'arrivée progressive de l'informatique dématérialisée dans l'industrie de la traduction est une réalité non virtuelle. La dématérialisation/virtualisation de l'environnement de travail du traducteur est synonyme de simplification, d'évolutivité, de mutualisation, de productivité et d'efficacité. Elle entraîne néanmoins une ultradépendance aux connexions permanentes à Internet et multiplie les risques de failles sécuritaires et les problèmes de confidentialité.

tilt.communications@wanadoo.fr

Anne-Marie ROBERT est titulaire d'un DESS de traduction professionnelle de I'ITIRI (Université de Strasbourg). Elle exerce à son compte depuis 1997 notamment en tant que traductrice technique spécialisée dans les nouvelles technologies, la TAO, la post-édition et la localisation informatique et multimédia (de l'anglais et de l'espagnol vers le français). Son engagement corporatiste se traduit par une implication à la fois dans la SFT, le syndicat national des traducteurs professionnels dont elle est la Vice-présidente, et dans la formation initiale et continue. Elle enseigne ses domaines de spécialité notamment en tant que PAST dans le cadre du Master "Métiers de la traduction " (Université de Provence) et est membre du Bureau de l'AFFUMT (Association française des formations universitaires aux métiers de la traduction).

\section{Webographie}

Boltran, http://www.boltran.com/home.seam, consulté le 27 mars 2011

Cloud Computing Journal, http://cloudcomputing.sys-con.com/, consulté le 27 mars 2011

Cloud Expo, http://cloudcomputingexpo.com/, consulté le 27 mars 2011

Crowdin, http://crowdin.net/, consulté le 27 mars 2011

GlobalSight, http://www.globalsight.com/, consulté le 27 mars 2011

Google Translator Toolkit, https://www.google.com/accounts/ServiceLogin?service=gtrans\&passive=true\&nui= 1 \&continue $=$ http://ranslate.google.com/toolkit?hl\%3Dfr\&followup=http://tran slate.google.com/toolkit?hl\%3Dfr\&hl=fr, consulté le 27 mars 2011

Lingotek, http://www.lingotek.com/, consulté le 27 mars 2011

Microsoft Office 365, http://office365.microsoft.com/en-US/online-services.aspx, consulté le 27 mars 2011

MySaas.fr, http://mysaas.fr/tag/saas/, consulté le 27 mars 2011 
SearchCloudComputing.com, http://searchcloudcomputing.techtarget.com/, consulté le 27 mars 2011

Translation Workspace, https://www.geoworkz.com/, consulté le 27 mars 2011

Wordbee Translator, http://www.wordbee.com/, consulté le 27 mars 2011

Wordfast Anywhere, http://www.wordfast.net/index.php?whichpage=anywhere\&lang=engb, consulté le 27 mars 2011

XTM Cloud, http://www.xtm-intl.com/xtmcloud, consulté le 27 mars 2011 